\title{
ERRATA
}

\section{Real-time detection of caspase- 2 activation in a single living HeLa cell during cisplatin-induced apoptosis}

\author{
Juqiang Lin \\ Zhihong Zhang \\ Jie Yang \\ Shaoqun Zeng \\ Bi-Feng Liu \\ Qingming Luo \\ [DOI: 10.1117/1.2219117]
}

This article was originally published online on 17 April 2006 with several technical errors. The key technical corrections that have been made to the article are as follows:

- Page 3: In the caption of Fig. 1, the phrase "(a 16-aminoacid peptide MHDQLTEEDEVDGSEL with a linker containing a caspase-2 recognition site sequence)" was changed to "(a 16-amino-acid peptide MHDQLTEEDEVDGGEL with a linker containing a caspase-3 recognition site sequence)".

- Page 3: In Sec. 3.2, the sentence beginning "The cisplatinuntreated group peaked at $585 \mathrm{~nm}$..." was changed to "The cisplatin-untreated group peaked at $580 \mathrm{~nm} . . . "$.

- Page 4: In Sec. 3.2, the phrase "we applied Western blot analysis of cellular extracts after 7 to $12 \mathrm{~h}$ of cisplatin treatment" was changed to "we applied Western blot analysis of cellular extracts after $12 \mathrm{~h}$ of cisplatin treatment."
- Page 4: Figs. 3(c) and 3(d) have been interchanged.

- Page 4: In the caption of Fig. 3, the phrase "7 to 12 h" has been changed to " $12 \mathrm{~h}$ " in both lines 2 and 3. In line 6 of the caption, "cisplatin-treated HeLa cells" has been changed to "cisplatin-untreated HeLa cells" and vice versa. Also, in lines 8 and 9 of the caption, "cisplatin-treated HeLa cells" has been changed to "cisplatin-untreated HeLa cells" and vice versa.

- Page 5: In the last line of Sec. 4 on page 5, the phrase "observed when CD2 or CD3 was excited at $430 \mathrm{~nm}$ " has been changed to "observed when $\mathrm{CD} 2$ or $\mathrm{CD} 3$ was excited at 433 nm."

All online versions of the article were corrected on 2 June 2006. The article appears correctly in print.

1083-3668/2006/11(4)/049801/1/\$22.00 @ 2006 SPIE 